\title{
Chordal automation towards high-order systems
}

\author{
A. Weinmann OVE, Life Senior Member IEEE
}

This article refers to practical and application-oriented design aspects arising in the field of chordal automation where stereographic projection is used. Several actions are discussed when high-order systems are involved. For average-order systems there exist elegant analytically concise methods to present and solve the problems. The chordal stability margin design is extended to pole assignment conditions, gradient analysis and actuation signal limits, also adequate for high-order systems.

Keywords: Chordal stability margin; pole assignment; gradients involved; actuation signal limitation

\section{Chordale Automation in Richtung Systeme höherer Ordnung.}

Dieser Beitrag befasst sich mit praktischen und anwendungsorientierten Aspekten der Auslegung im Bereich der chordalen Automation beim Einsatz von stereographischer Projektion. Es werden mehrere Prozesse behandelt, an denen Systeme höherer Ordnung beteiligt sind. Für Systeme durchschnittlicher Ordnung gibt es elegante, analytisch prägnante Methoden, die Probleme darzustellen und zu lösen. Die Auslegung des chordalen Stabilitätsrandes wird ausgeweitet auf die Bedingungen der Eigenwertvorgabe, die Analyse mit Gradienten und die Begrenzung der Stellgröße; Methoden, welche auch für Systeme höherer Ordnung anwendbar sind.

Schlüsse/wörter: Chordaler Stabilitätsrand; Eigenwertvorgabe; Gradientennutzung; Stellgrößenbegrenzung

Received June 14, 2017, accepted July 3, 2017, published online July 20, 2017

(C) The Author(s) 2017. This article is published with open access at Springerlink.com

\section{Introduction}

An automatic control setup, given as a single-loop linear control system with a two-valued input and output, is considered in detail, see Fig. 1. The dynamic property results from the closed-loop poles $s$ of the transfer functions, i.e., of $1+G(s) K(s)=0$ or $G(s)=-K(s)^{-1}$. If $s=j \omega$ was fulfilled by any solution, the system would stay in an unwanted steady-state oscillation (if there was no otherwise solution $\Re$ Re $>0$ ). That is, a necessary condition, to simplify matters, reads as $G(j \omega) \neq-K^{-1}(j \omega), G(j \omega)$ and $-K(j \omega)^{-1}$ must be distinctly separated, $\min _{\omega}\left|G(j \omega)+K^{-1}(j \omega)\right|>0$. This makes clear that an interrelation of $G$ and the inverse $K$ is responsible for the closed-loop performance. To consider inverses of proper transfer functions is not common with reference to frequency plots routing to infinity. But the stereographic projections keeps it in the usual range.

From Fig. 1 one has

$$
\begin{aligned}
& \left.\frac{y-y_{\text {ref }}}{y_{\text {ref }}}\right|_{w_{d}=0}=\frac{1}{1+G K}=S \text { Sensitivity } \\
& \left.\frac{u}{w_{d}}\right|_{y_{\text {ref }}=0}=S \text { Sensitivity, Actuation caused by disturbance } \\
& \left.\frac{y}{y_{\text {ref }}}\right|_{w_{d}=0}=\frac{G K}{1+G K}=T=G K S
\end{aligned}
$$

Target Transfer function, output/reference

$\left.\frac{y}{W_{d}}\right|_{y_{\text {ref }}=0}=\frac{G}{1+G K}=G S$ Output caused by disturbance

$\frac{u}{y_{\text {ref }}} \operatorname{l}_{W_{d}=0}=\frac{K}{1+G K}=K S \quad$ Actuation caused by reference

$$
\left(\begin{array}{l}
y \\
u
\end{array}\right)=\left(\begin{array}{cc}
G S K & G S \\
K S & S
\end{array}\right)\left(\begin{array}{l}
y_{\text {ref }} \\
w_{d}
\end{array}\right) \triangleq \mathbf{M}\left(\begin{array}{l}
y_{\text {ref }} \\
w_{d}
\end{array}\right) \text {. }
$$

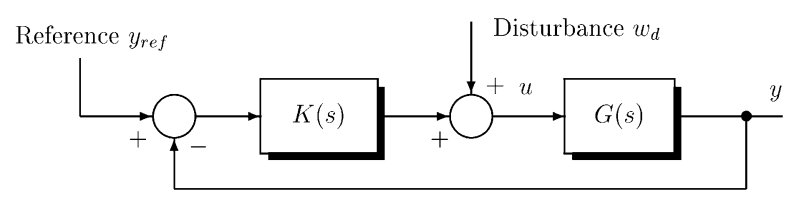

Fig. 1. Control system with controller $K$ and (uncertain) plant $G$

Now, the Riemann sphere is considered. In spite of the analytical effort, relations on a sphere are well suited for requirements in automatic control $[2,3]$. The Riemann sphere is situated at the complex plane of frequency plots with its south pole at the origin. Using stereographic projection onto the Riemann sphere, two points $G_{1}$ and $G_{2}$ of a frequency plot in the complex plane are mapped to $\mathbf{g}_{1}$ and $\mathbf{g}_{2}$ on the Riemann sphere via an arrow pointing to the north pole, i.e. map $G \mapsto \mathbf{g}$ corresponds with $\mathbf{g}=\left[\Re e G, \Im m G,|G|^{2} /\left(1+|G|^{2}\right)\right]$.

The straight distance inside the sphere, Fig. 2, results from basic algebra

$$
\left\|\mathbf{g}_{1}-\mathbf{g}_{2}\right\|_{F}=\frac{1}{\sqrt{1+\left|G_{1}\right|^{2}} \sqrt{1+\left|G_{2}\right|^{2}}}\left|G_{1}-G_{2}\right| .
$$

For automatic control purposes, the chordal distance $\rho[G, K]$ is the inverse of the maximum singular value of $\mathbf{M}$ (conditionally weighted by $w_{i k}$ ).

Weinmann, Alexander, Institute of Automation and Control, Vienna University of

Technology, Gusshausstrasse 27-29/376, 1040 Vienna, Austria

(E-mail: weinmann@acin.tuwien.ac.at) 


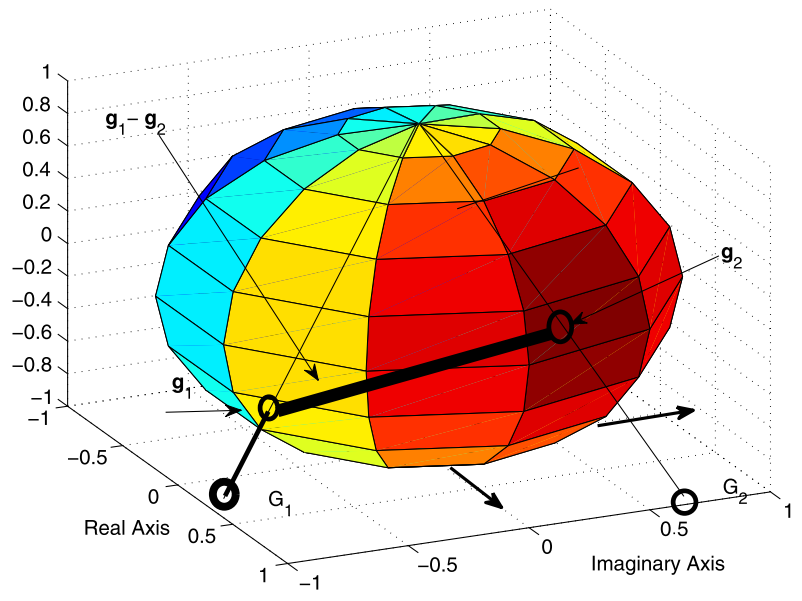

Fig. 2. Chordal distance with norm $\left\|g_{1}-g_{2}\right\|_{F}$ at the Riemann sphere

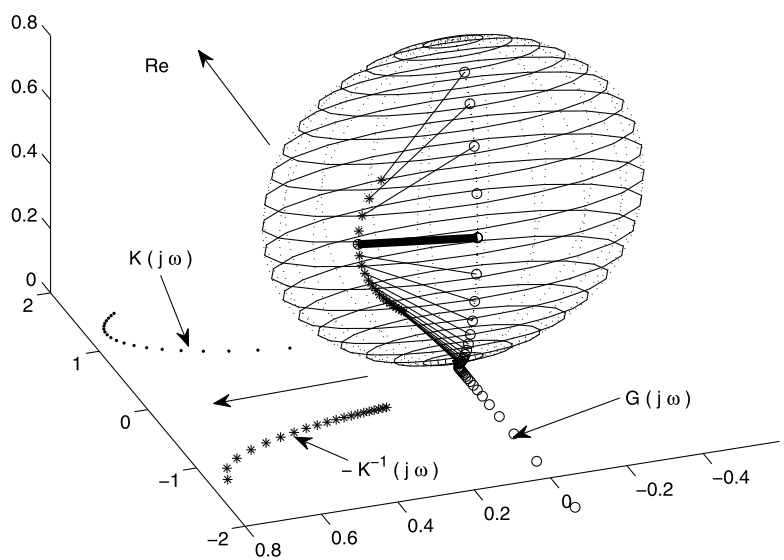

Fig. 3. Minimum chordal distance $b_{G K}$ between $G$ and $-K^{-1}$ as a bold face line

$$
\begin{aligned}
\rho[G, K] & \triangleq\left\{\sigma_{\max }[\mathbf{M}]\right\}^{-1} \\
& =\left\{\sigma_{\max }\left[\left(\begin{array}{cc}
T & G S \\
S K & S
\end{array}\right)\right]\right\}^{-1} \\
& =\left\{\sigma_{\max }\left[\left(\begin{array}{c}
G \\
1
\end{array}\right)(1+K G)^{-1}(K 1)\right]\right\}^{-1} .
\end{aligned}
$$

Comparing Eq. (7) and Eq. (45), the extension of Eq. (8), and Eq. (36) is important, especially the correspondences $G_{1}, G_{2}$ and $-K^{-1}, G$, respectively. They are used for purpose in automatic control

$$
\rho[G, K]=\delta\left[-K^{-1}, G\right] .
$$

The chordal stability margin is defined by the minimum versus frequency

$$
b_{G K} \triangleq \inf _{\omega} \rho[G(j \omega), K(j \omega)] .
$$

Presupposing stability of the nominal system of Fig. 1, a given family of uncertain (perturbed) plants $G_{p}$ is robustly stable if [1-3]

$$
b_{G K}>r_{G} \triangleq \max _{\omega} \delta\left[G_{p}(j \omega), G(j \omega)\right] .
$$

This relation has been slightly simplified. The chordal stability margin must be bigger than the chordal uncertainty radius $r_{G}$. Using the
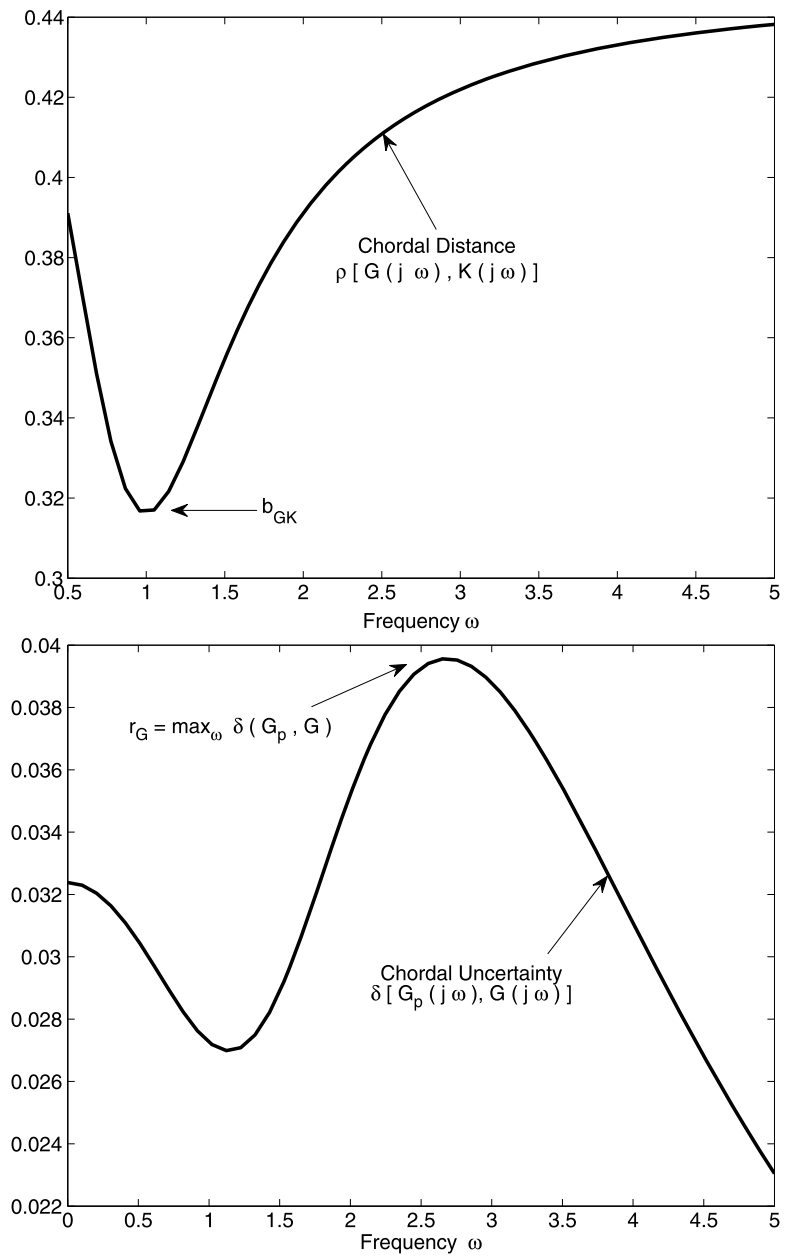

Fig. 4. Cartesian plot of the stability margin (upper figure) and uncertainty versus frequency (lower figure)

definitions Eq. (46) and Eq. (38) of the Appendix A,

$$
\begin{aligned}
& b_{G K} \triangleq \min _{\omega} \frac{1}{\sqrt{1+\left(\left|\frac{G(-s)-K(s)}{1+G(s) K(s)}\right|_{s=j \omega}\right)^{2}}} \\
& >r_{G} \triangleq \max _{\omega} \frac{1}{\sqrt{1+\left|\frac{G_{p}(s)-G(s)}{1+G_{p}(s) G(-s)}\right|_{s=j \omega}^{(-2)}}} .
\end{aligned}
$$

For illustration, an example for $K(s)=(1+2 s) /(2+s), G(s)=1 / s^{2}$ is given in Figs. 3 and 4.

Now, the main part of this article aims at application-oriented problems characterized especially by high-order systems. The chordal stability margin is extended by a gradient method. An optimization uses eigenvalue assignment and actuation effort conditions. The unstructured chordal stability margin is also combined with the unstructured design method using the inverse trace of system matrix in state space.

\section{Gradient of the chordal stability margin $b_{G K}$}

Define the polynomial setup of the controller $K(j \omega)=\frac{Z(j \omega)}{N(j \omega)}, N(j \omega)=$ $\mathbf{b}^{T} \boldsymbol{\omega}, Z(j \omega)=\mathbf{a}^{T} \boldsymbol{\omega}, \boldsymbol{\omega} \triangleq\left(1 j \omega-\omega^{2}-j \omega^{3} \ldots\right)^{T} \in \mathcal{C}^{n}, \omega \in \mathcal{R}^{1}, \boldsymbol{\omega}^{H} \equiv$ $\omega^{\star T}$

$$
\boldsymbol{\Omega} \triangleq \boldsymbol{\omega} \boldsymbol{\omega}^{H}=\left(\begin{array}{ccc}
1 & -\omega j & -\omega^{2} \\
\omega j & \omega^{2} & -\omega^{3} j \\
-\omega^{2} & \omega^{3} j & \omega^{4}
\end{array}\right)
$$


$\boldsymbol{\Omega}_{r} \triangleq \Re e \boldsymbol{\Omega}, \boldsymbol{\Omega}_{i} \triangleq \Im m \boldsymbol{\Omega},|N|^{2}=\mathbf{b}^{T} \boldsymbol{\Omega} \mathbf{b},|Z|^{2}=\mathbf{a}^{T} \mathbf{\Omega} \mathbf{a}$

$$
\begin{aligned}
b_{G K} & \stackrel{(10)}{=} \min _{\omega} \rho[G, K] \\
& =\min _{\omega} \frac{1}{\sqrt{1+|G|^{2}} \sqrt{1+\left|K^{-1}\right|^{2}}}\left|K^{-1}+G\right| \\
& =\min _{\omega} \frac{U_{1} U_{2}}{\sqrt{1+|G|^{2}}}
\end{aligned}
$$

where

$$
\begin{aligned}
U_{1} & \triangleq\left|-K^{-1}+G\right| \\
& =\sqrt{\left(\frac{-\mathbf{a}^{T} \boldsymbol{\Omega}_{r} \mathbf{b}}{\mathbf{a}^{T} \mathbf{\Omega} \mathbf{a}}+\Re e G\right)^{2}+\left(\frac{-\mathbf{a}^{T} \mathbf{\Omega}_{i} \mathbf{b}}{\mathbf{a}^{T} \mathbf{\Omega} \mathbf{a}}+\Im m G\right)^{2}} \\
U_{2} & \triangleq \frac{1}{\sqrt{1+\left|K^{-1}\right|^{2}}}=\frac{1}{\sqrt{1+\frac{\mathbf{b}^{T} \mathbf{\Omega} \mathbf{b}}{\mathbf{a}^{T} \mathbf{a}}}} .
\end{aligned}
$$

With $b_{i}$ as one of the coefficients of $\mathbf{b}$ in the denominator polynomial, responsible for the poles of the controller, and $\mathbf{e}_{i}$ the unit vector

$$
\begin{gathered}
\frac{\partial U_{1}}{\partial b_{i}}=\frac{\mathbf{a}^{T}}{U_{1} \mathbf{a}^{T} \boldsymbol{\Omega a}}\left[\left(-\frac{\mathbf{a}^{T} \boldsymbol{\Omega}_{r} \mathbf{b}}{\mathbf{a}^{T} \boldsymbol{\Omega a}}-\Re e G\right) \boldsymbol{\Omega}_{r}\right. \\
\left.+\left(-\frac{\mathbf{a}^{T} \boldsymbol{\Omega}_{i} \mathbf{b}}{\mathbf{a}^{T} \boldsymbol{\Omega a}}+\Im m G\right) \boldsymbol{\Omega}_{i}\right] \mathbf{e}_{i} \\
\frac{\partial U_{2}}{\partial b_{i}}=-U_{2}^{3} \frac{\mathbf{b}^{T} \boldsymbol{\Omega} \mathbf{e}_{i}}{\mathbf{a}^{T} \boldsymbol{\Omega a}} \\
\frac{\partial \rho[G, K]}{\partial b_{i}} \stackrel{(14)}{=} \frac{1}{\sqrt{1+|G|^{2}}}\left(U_{2} \frac{\partial U_{1}}{\partial b_{i}}+U_{1} \frac{\partial U_{2}}{\partial b_{i}}\right) .
\end{gathered}
$$

Changing the order of operations one has

$$
\begin{aligned}
\frac{\partial b_{G K}}{\partial b_{i}} & =\frac{\partial}{\partial b_{i}}\left(\inf _{\omega} \rho[G, K]\right) \\
& =\inf _{\omega}\left(\frac{\partial \rho[G, K]}{\partial b_{i}}\right) \\
& =\inf _{\omega}\left[\frac{U_{2} \frac{\partial U_{1}}{\partial b_{i}}+U_{1} \frac{\partial U_{2}}{\partial b_{i}}}{\sqrt{1+|G|^{2}}}\right] .
\end{aligned}
$$

An extension to vector-valued gradients $\frac{\partial}{\partial \mathbf{b}}$ or $\frac{\partial}{\partial \mathbf{a}}$ for uncertain coefficients of $G$ or $K$ can easily be stated, irrespective of the system order.

\section{Eigenvalue assignment as a condition}

Consider a controller $K$ which order is selected intentionally higher, using a surplus of e.g. $c_{1}$ and $c_{2}$ of $K(s)$. Assume a first-order plant and a second-order controller

$$
G(s)=\frac{g_{1}}{g_{2}+g_{s} s}, \quad K(s)=\frac{c_{0}+c_{1} s+c_{2} s^{2}}{n_{0}+n_{1} s+n_{2} s^{2}}
$$

and assigned poles of the control system

$$
a_{0}+a_{1} s+\cdots+a_{3} s^{3} \quad(-1.04 ;-1.47 \pm j 4.5) .
$$

Then the system is underdetermined referring to the parameters $c_{1}$ and $c_{2}$. They can be arbitrarily chosen without affecting the eigenvalue assignment. The task is

$$
\begin{aligned}
& b_{G K}\left(a_{i}, n_{i}, g_{i}, c_{i}\right) \rightarrow \max _{c_{1}, c_{2}} \text { subject to } \\
& \left(\begin{array}{cccc}
g_{2} & 0 & 0 & g_{1} \\
g_{3} & g_{2} & 0 & 0 \\
0 & g_{3} & g_{2} & 0 \\
0 & 0 & g_{3} & 0
\end{array}\right)\left(\begin{array}{l}
n_{0} \\
n_{1} \\
n_{2} \\
c_{0}
\end{array}\right)=\left(\begin{array}{c}
a_{0} \\
a_{1}-g_{1} c_{1} \\
a_{2}-g_{1} c_{2} \\
a_{3}
\end{array}\right) .
\end{aligned}
$$

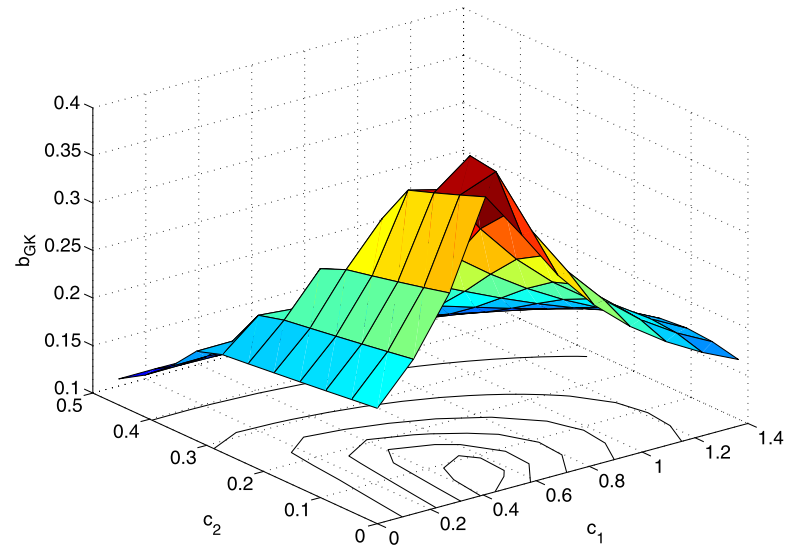

Fig. 5. Maximizing $b_{G K}$ with conditions

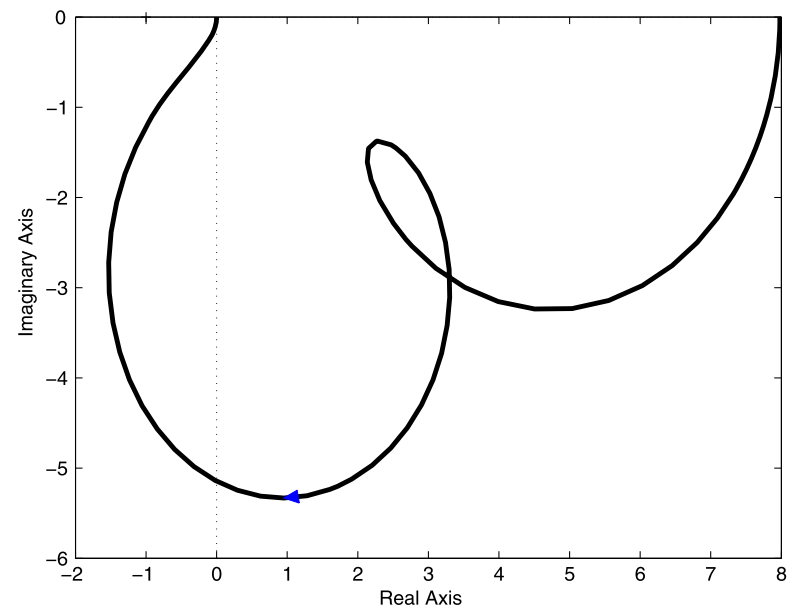

Fig. 6. Optimal open-loop frequency characteristic $G(j \omega) K(j \omega)$

The optimum controller results as

$$
K=\frac{0.09091 s^{2}+0.5455 s+0.7109}{0.1429 s^{2}+0.141 s+1.336} \text { and } b_{G K}=0.421 \text {, }
$$

portrayed in Figs. 5 and 6 .

\section{Valuating constraints}

An important issue is valuating constraints, e.g. the maximum actuation signal $u_{m}$ arising from a reference step function. What is the design process? In Fig. 7 lines of given constant values $b_{G K}$ are repeated in the basis plane $c_{1}, c_{2}$. A plane of resulting $u_{m}$ is depicted. Above one of these lines of constant $b_{G K}$, a single line of values of $u_{m}$ is plotted as a thick line. This line demonstrates $u_{m}\left(c_{1}, c_{2}\right)$ with no influence on the implemented eigenvalue assignment and chosen chordal stability margin $b_{G K}$.

\section{Structured rectangular uncertainty versus chordal} uncertainty

The question arises what is the chordal uncertainty measure $\delta\left[G_{p}, G\right]$ for a given structured rectangular uncertainty in $G_{p}=\frac{2 s+b_{z}}{s^{2}+3 s+a_{N}}$; $a_{N}=5, b_{Z}=4, K(s)=\frac{s+2}{20 s+1}$, with parameters $a_{N}, b_{Z}$ uncertain less than a certain amount, see Fig. 8.

Another question is: Which rectangular uncertainty is equivalent to a chordal one. Which part of the rectangular parameters is included if e.g. $\delta\left[G, G_{p}\right]<0.03$ is given. The result is depicted in Fig. 9 . 


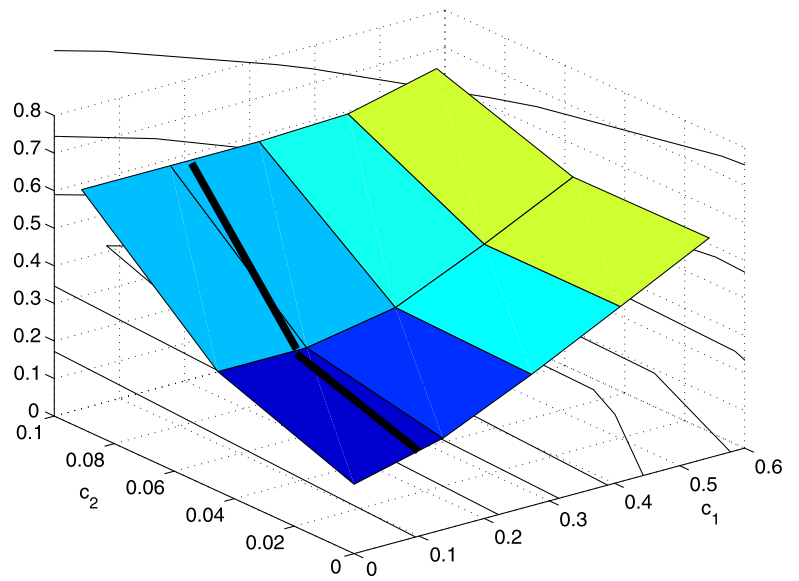

Fig. 7. $u_{m}\left(c_{1}, c_{2}\right)$ where the eigenvalue assignment and $b_{G K}$ are untouched

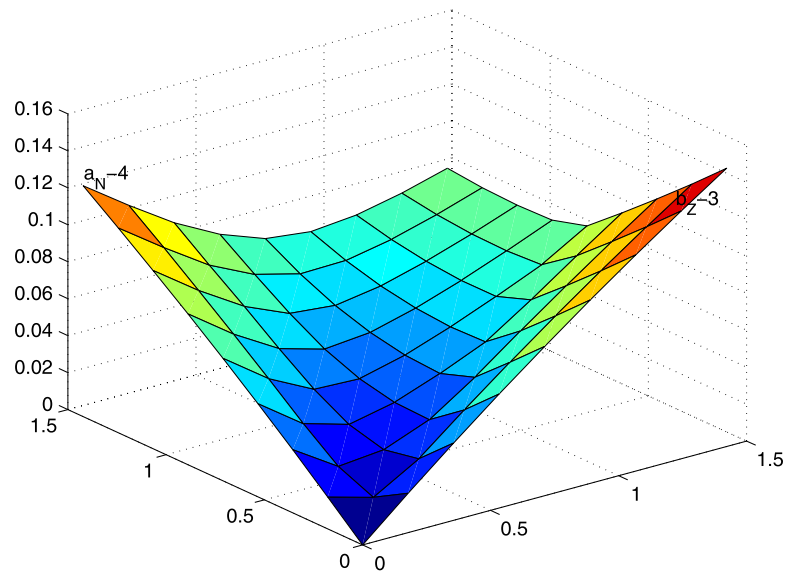

Fig. 8. $\delta\left[G_{p}, G\right]$ for the rectangular uncertainty

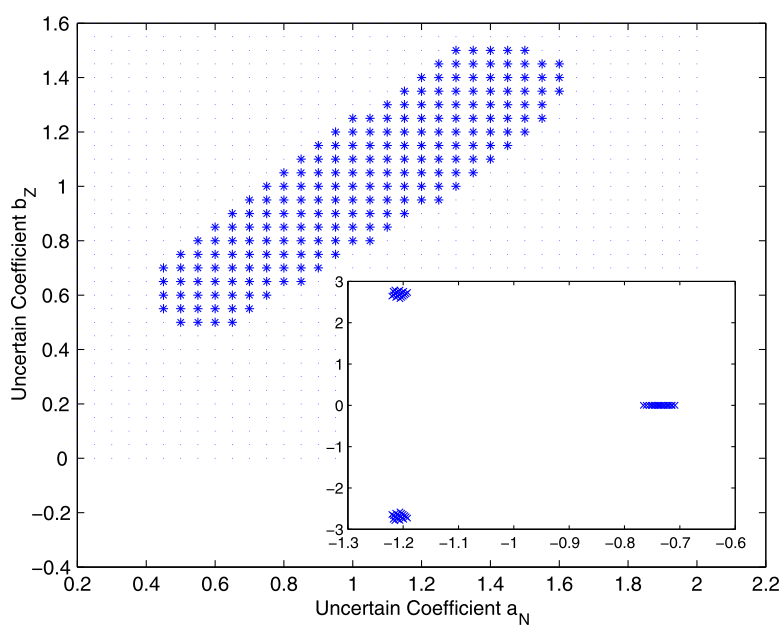

Fig. 9. Range of coefficients for $r_{G}=\delta\left[G_{p}, G\right]<0.03$

For illustration purpose the set of eigenvalues of the closed-loop system are included in this figure when a so-called uncertainty walk is executed along the border of the chordal uncertainty.

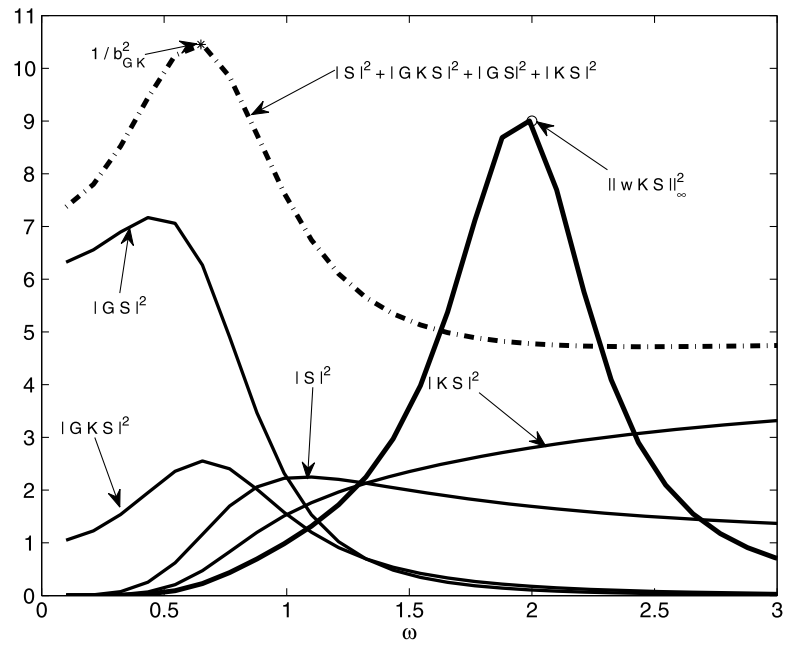

Fig. 10. Elements of $N$ of $b_{G K}$ and $H_{\infty}$, limit. $K(s)=(2 s+1) /$ $(s+2.5), G(s)=1 / s^{2}$

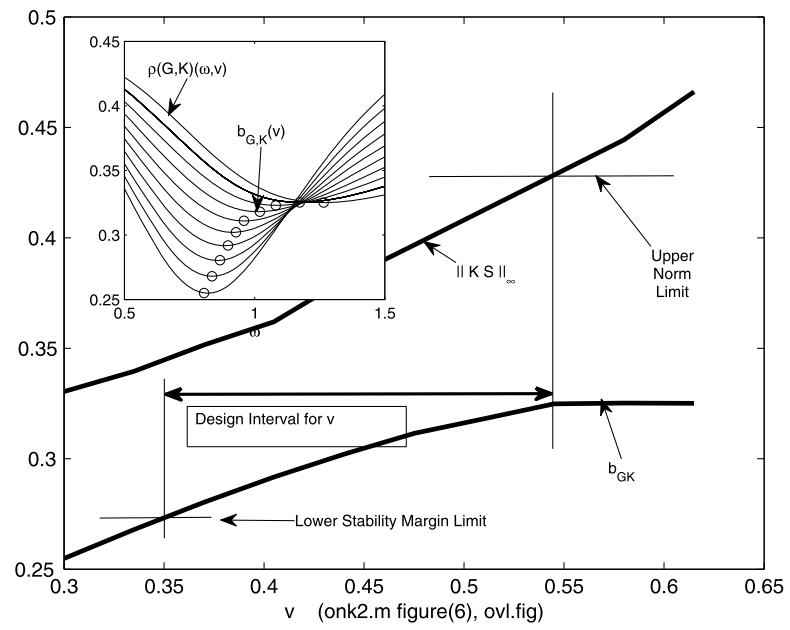

Fig. 11. Stability margin $b_{G K}$ for $K=(1+2 v s) /(s+2)$ including a bound for $\|K S\|_{\infty}$

\section{Graphically supported design}

Figure 10 is derived from Eq. (58). The function $N$ is depicted versus frequency before having selected the minimum. The areas between the function and the abscissa are $\int_{0}^{\infty}(\cdot)^{*}(\cdot) d \omega$. In the figure, 6.3 stands for $|G S|^{2}$ and 2.5 for $|G K S|^{2}$, for the outputs caused by disturbance and reference in the steady state, respectively. These values represent the root mean square energy content of the signals. According to Parseval Theorem they correspond to the integral of squared impulse response. Usual requirements are increasing $b_{G K}$, observing all the actuations and selected limits. Besides, also the squared initial values can be observed if the frequency axis is scaled up to higher values; the initial actuation signal, 4 and 1, from $\mathrm{KS}$ and $\mathrm{S}$, caused by reference and disturbance, respectively. When changing parameters during the design process, special limits, e.g. $\|W K S\|_{\infty}$, can be taken into consideration.

The influence of a varying design parameter $v$ is shown in Fig. 11. A subplot demonstrates the frequency-dependent $\rho(G, K)(\omega ; v)$ and the local minimum in $b_{G K}$. 
An alternative could be: Varying $k_{i}$, elements of $K$, from $1 / b_{G K}^{2} \rightarrow$ $\min _{k_{i}}$ from Eq. (48) one finds

$$
1+\left\|\frac{G(-s)-K}{1+G K}\right\|_{\infty}^{2}+\eta\|K S\|_{\infty}^{2} \rightarrow \min _{k_{i}}
$$

Then, $b_{G K}$ and $\|K S\|_{\infty}$ are displayed having an eye on the limits.

\section{Chordal stability margin for holistic eigenvalue assignment}

The trace of the negative inverse closed-loop coefficient matrix in state-space system design is the sum of inverse poles [4]. Shifting the poles to the left as much as possible

$$
-\sum_{i} 1 / \lambda_{i}[\mathbf{A}+\mathbf{B K}]=-\operatorname{tr}\left\{\mathbf{A}_{c l}^{-1}\right\} \rightarrow \min _{\mathbf{K}}
$$

is required, conditionally augmented by the norm of $\mathbf{K}$. Now, when $b_{G K}$ is included

$$
\frac{1}{-\operatorname{tr}\left[\mathbf{A}_{c l}^{-1}\right]}+b_{G K} \rightarrow \max _{\mathbf{K}}
$$

has to be executed. Figure 12 depicts the result with an additional condition $c_{2}>2$.

Open Access This article is distributed under the terms of the Creative Commons Attribution 4.0 International License (http://creativecommons.org/ licenses/by/4.0/), which permits unrestricted use, distribution, and reproduction in any medium, provided you give appropriate credit to the original author(s) and the source, provide a link to the Creative Commons license, and indicate if changes were made.

\section{Appendix A}

This appendix is considered as a clearly arranged presentation of interrelations which might be confound with each other, and to get familiar with the intermediate derivations, even though they are known in the literature [1-3],

\section{A.1. Singular values}

The singular value obeys $\sigma[\mathbf{G}] \triangleq+\sqrt{\lambda\left[\mathbf{G}^{H} \mathbf{G}\right]}$ and its maximum $\sigma_{\max }[\mathbf{G}]=+\sqrt{\lambda_{\max }\left[\mathbf{G}^{H} \mathbf{G}\right]} \equiv\|\mathbf{G}\|_{s}$. The $H_{\infty}$ norm is $\|\mathbf{G}(s)\|_{\infty} \triangleq$ $\sup _{\omega} \sigma_{\max }[\mathbf{G}(j \omega)]$. The maximum singular value is a generalization of the absolute value of scalars for matrices.

For any matrix $\mathbf{X}$ or a special vector $\mathbf{X}=(a, b)^{T}$ one has $\mathbf{X}^{H} \mathbf{X}=$ $(a, b)^{\star}(a, b)^{T}=\left(a^{*} a+b^{*} b\right)$ and

$$
\begin{aligned}
\sigma_{\max }[\mathbf{X Y}] & =\sqrt{\lambda_{\max }\left[(\mathbf{X Y})^{H} \mathbf{X Y}\right]}=\sqrt{\lambda_{\max }\left[\mathbf{Y}^{H} \mathbf{X}^{H} \mathbf{X Y}\right]} \\
& =\sqrt{\lambda_{\max }\left[\mathbf{Y}^{H}\left(\mathbf{X}^{H} \mathbf{X}\right)^{0.5}\left(\mathbf{X}^{H} \mathbf{X}\right)^{0.5} \mathbf{Y}\right]} \\
& =\sigma_{\max }\left[\left(\mathbf{X}^{H} \mathbf{X}\right)^{0.5} \mathbf{Y}\right] \\
& =\sigma_{\max }\left[\left(a^{\star} a+b^{\star} b\right)^{0.5} \mathbf{Y}\right]=\sigma_{\max }\left[\sqrt{a^{\star} a+b^{\star} b} \mathbf{Y}\right] .
\end{aligned}
$$

\section{A.2. Chordal uncertainty measurement $\delta\left[G_{p}, G\right]$}

Note that $p^{*}(j \omega)=\left.p(-s)\right|_{s=j \omega}$ and $\left.a(s) \cdot a(-s)\right|_{s=j \omega}=\mathbf{a}^{T} \boldsymbol{\omega} \cdot \mathbf{a}^{T} \boldsymbol{\omega}^{*} \stackrel{(13)}{=}$ $\mathbf{a}^{T} \boldsymbol{\Omega} \mathbf{a}$. Note also $\left.G^{*} \triangleq G(-s)\right|_{s=j \omega}$ (for functions rational real $P^{*}(j \omega)=$ $\operatorname{conj}\left(P(j \omega)=\left.P(-s)\right|_{s=j \omega}\right)$.

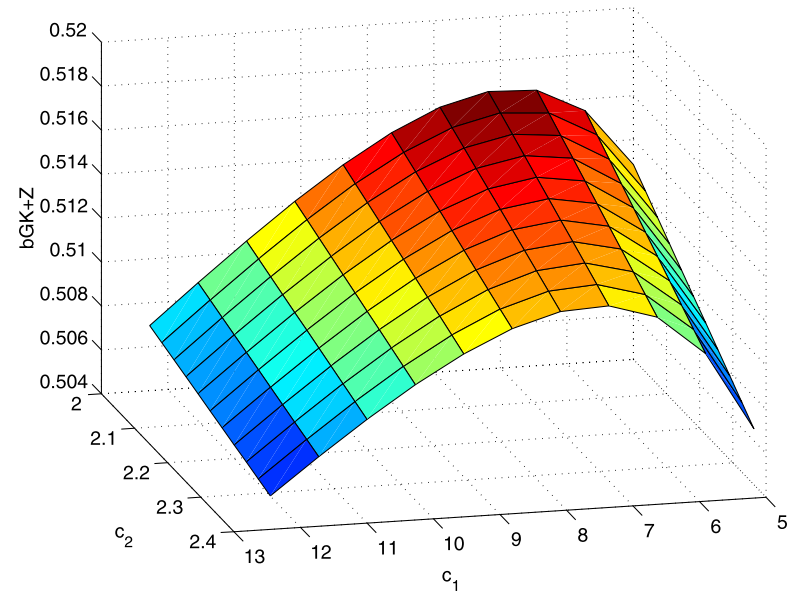

Fig. 12. Combining $b_{G K}$ with holistic sum of eigenvalues

\section{Conclusion}

The presented methods are intended to demonstrate high-order control systems and graphically supported design tools, adequate for applications. The methods are selected of simple structure in order to simplify the access.

\section{Acknowledgements}

Open access funding provided by TU Wien (TUW).

There is the rather strange definition of the distance of two transfer functions where each one is a function of $s=j \omega$, but it turns out similar Eq. (7)

$$
\begin{aligned}
& \left.\delta\left[G_{1}(j \omega)\right), G_{2}(j \omega)\right] \\
& =\left.\frac{1}{\sqrt{1+\left|\frac{G_{1}-G_{2}}{1+G_{1} G_{2}(-s)}\right|^{(-2)}}}\right|_{s=j \omega}=\left.\frac{1}{\sqrt{1+\left|\frac{1+G_{1} G_{2}^{*}}{G_{1}-G_{2}}\right|^{2}}}\right|_{s=j \omega} \\
& =\left.\frac{1}{\sqrt{\frac{\left(G_{1}-G_{2}\right)\left(G_{1}-G_{2}\right)^{*}+\left(1+G_{1} G_{2}^{\star}\right)\left(1+G_{1}^{\star} G_{2}\right)}{\left|G_{1}-G_{2}\right|^{2}}}}\right|_{S=j \omega} \\
& =\left.\frac{1}{\sqrt{\frac{\left|G_{1}\right|^{2}+\left|G_{2}\right|^{2}+1+\left|G_{1}\right|^{2}\left|G_{2}\right|^{2}}{\left|G_{1}-G_{2}\right|^{2}}}}\right|_{s=j \omega} \\
& =\left.\frac{\left|G_{1}-G_{2}\right|}{\sqrt{\left(1+\left|G_{1}\right|^{2}\right)\left(1+\left|G_{2}\right|^{2}\right)}}\right|_{S=j \omega} \text {. }
\end{aligned}
$$

For the case of uncertain plant $G_{1}=G_{p}$ and $G_{2}=G$.

For $G_{1}$ close to $G_{2}$ and for small uncertainty, the 1 in Eq. (33) can be neglected

$$
\max _{\omega} \delta\left[G_{p}(s), G(s)\right]=\max _{\omega} \delta\left[G_{p}(j \omega), G(j \omega)\right]=\left\|\frac{G_{p}-G}{1+G_{p} G(-s)}\right\|_{\infty} .
$$

For the nominal $G$, the family of perturbed $G_{p}$ and $\delta\left[G(s), G_{p}(s)\right]$ one has

$$
\max _{\omega} \delta\left[G_{p}(j \omega), G(j \omega)\right]=\left.\max _{\omega} \frac{1}{\sqrt{1+\left|\frac{G_{p}-G}{1+G_{p} G(-s)}\right|(-2)}}\right|_{s=j \omega} \triangleq r_{G} .
$$

A.3. Chordal distance $\rho[G, K]$ and stability margin

$$
\rho[G, K] \stackrel{(8)}{=}\left\{\sigma_{\max }\left[\left(\begin{array}{l}
G \\
1
\end{array}\right)(1+K G)^{-1}(K 1)\right]\right\}^{-1}
$$




$$
\begin{aligned}
& \stackrel{\text { (32) }}{=}\left\{\sigma_{\max }\left[\sqrt{(G \quad 1)^{\star}\left(\begin{array}{c}
G \\
1
\end{array}\right)}(1+K G)^{-1}(K \quad 1)\right]\right\}^{-1} \\
& =\left\{\sigma_{\max }\left[\sqrt{1+G^{\star} G}(1+K G)^{-1}(K 1)\right]\right\}^{-1} \\
& =\left\{\left|\sqrt{1+G^{\star} G}(1+K G)^{-1} \sqrt{1+K^{\star} K}\right|\right\}^{-1} \\
& =\left\{\left|\sqrt{1+G^{\star} G}(1+K G)^{-1} K \frac{1}{K} \sqrt{1+K^{\star} K}\right|\right\}^{-1} \\
& =\left\{\left|\sqrt{1+G^{\star} G}\left(K^{-1}+G\right)^{-1} \sqrt{\frac{1}{K} \frac{1}{K^{*}}+1}\right|\right\}^{-1} \\
\rho[G, K] & =\frac{\left|-\left(-K^{-1}\right)+G\right|}{\sqrt{1+|G|^{2}} \sqrt{1+\left|K^{-1}\right|^{2}}} \stackrel{(36)}{=} \delta\left[G, K^{-1}\right] .
\end{aligned}
$$

Further correspondencies referring to $b_{G K}$ are given as follows. For $G_{1}=-K^{-1}$ and $G_{2}=G$ in Eq. (36), $b_{G K}$ is defined as the smallest chordal distance between $G$ und $-K^{-1}$

$$
\begin{aligned}
b_{G K} & \triangleq \min _{\omega} \delta\left[-K^{1}, G\right] \triangleq \min _{\omega}\left\{1+\left|\frac{-K^{-1}-G}{1-K^{-1} G(-s)}\right|_{\omega}^{-2}\right\}^{-1 / 2} \\
& =\min _{\omega}\left\{1+\left|\frac{-1-G K}{K-G(-s)}\right|_{\omega}^{-2}\right\}^{-1 / 2} \text { and with inversion } \\
& =\left\{1+\|\left.\frac{G(-s)-K}{1+G K}\right|_{\infty} ^{2}\right\}^{-1 / 2},
\end{aligned}
$$

where $\min _{\omega}\left|G^{-1}\right|=\max _{\omega}|G|=\|G\|_{\infty}$ was used.

We start a new derivation with Eq. (45) and we multiply numerator and denominator with $|K|$

$$
\begin{aligned}
\rho[G, K] \stackrel{(45)}{=} \frac{\left(\left|K^{-1}+G\right|\right)|K|}{\sqrt{1+|G|^{2}} \sqrt{1+\left|K^{-1}\right|^{2}}|K|} \\
=\frac{\sqrt{\left(K^{-1}+G\right)\left(K^{-1 \star}+G^{\star}\right)} \sqrt{K K^{\star}}}{\sqrt{1+|G|^{2}} \sqrt{1+|K|^{2}}} \\
=\frac{\sqrt{(1+G K)\left(1+G^{\star} K^{\star}\right)}}{\sqrt{1+|G|^{2}} \sqrt{1+|K|^{2}}} \\
=\frac{|1+G K|}{\sqrt{1+|G K|^{2}+|G|^{2}+|K|^{2}}} .
\end{aligned}
$$

\section{Author}

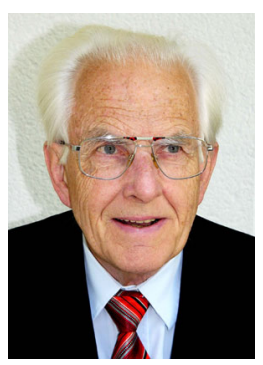

\section{Alexander Weinmann}

Born in Vienna in 1937, 1960: Dipl.-Ing. 1962: Dr. techn., 1966: Habilitation "Elektrische Antriebs- und Regelungstechnik", 19682005: O. Univ.-Prof. "Elektrische Regelungsund Steuerungstechnik" at Vienna University of Technology. Head of the Institute of Automatic Control 1968-2004. Long-term Dean of the Faculty of Electrical Engineering, coinitiating their foundation. Managing head of the former computing center of the entire university, chair of several commissions for academic administration, tuition and curriculum development.
Alternatively, from Eqs. (12), (46) and (47) it follows $b_{G K} \triangleq$ $\min _{\omega} \frac{1}{\sqrt{N}}$ and

$$
\begin{aligned}
N & =\frac{|1+G K|^{2}+|G(-s)-K|}{|1+G K|^{2}} \\
& =\frac{\left[1+(G K)^{\star}\right][1+(G K)]+\left[G(-s)^{\star}-K^{\star}\right][G(-s)-K]}{|1+G K|^{2}} \\
& \stackrel{s=j \omega \omega}{=} \frac{1+(G K)^{\star}+(G K)+(G K)^{\star}(G K)+G G^{\star}-K^{\star} G^{\star}-G K+K^{\star} K}{|1+G K|^{2}}
\end{aligned}
$$

$N=\frac{1+(G K)^{\star}(G K)+G G^{\star}+K^{\star} K}{|1+G K|^{2}}=\frac{1+|G K|^{2}+|G|^{2}+|K|^{2}}{|1+G K|^{2}}$.

The chordal distance $p[G, K]$ of Eq. (52) equals $1 / \sqrt{N}$ of Eq. (56).

$$
\begin{aligned}
b_{G K} & =\min _{\omega} \frac{1}{\sqrt{N}}=\frac{1}{\sup _{\omega} \sqrt{N}}=\frac{1}{\sup _{\omega} \frac{\sqrt{1+|G K|^{2}+|G|^{2}+|K|^{2}}}{|1+G K|}} \\
& =\frac{1}{\sup _{\omega} \sqrt{|S|^{2}+|T|^{2}+|G S|^{2}+|K S|^{2}}} .
\end{aligned}
$$

Take notice of the different interpretations of $b_{G K}$. The origin resulting of automatic control Eq. (39); the chordal distance in Eq. (45); the minimum value in frequency domain in Eq. (12); an expression using $H_{\infty}$-norm Eq. (48) and the graphical correspondence using all the components of $\mathbf{M}$ in Eq. (58)

\section{References}

1. El-Sakkary, A. K. (1989): Estimating robustness on the Riemann sphere. Int. J. Control, $49,561-567$.

2. Qiu, L., Zhou, K. (2013): Preclassical tools in postmodern control. IEEE Control Syst. Mag., 33(4), 26-38.

3. Vinnicombe, G. (2001): Uncertainty and feedback: $H_{\infty}$ loop shaping and the $v$-gap metric. London: Imperial College Press.

4. Weinmann, A. (2001): Control system design based on holistic eigenvalue allocation. E\&I, Elektrotech. Inf.tech., 118, 167-173.

He worked on various topics related to optimal control systems and design, identification, robustness, observers. Single author (without co-authorship) of roughly 140 scientific papers and seven books in several editions. 18 patents.

Consultant of ELIN with actual project responsibility, and other enterprises, up to 2006.

Editor and associate editor of several journals. Honor member OVE and ASCS. Among others: Awards Karoline and Guido Krafft, Kardinal Innitzer, Golden Stefan Medal of OVE. 